\title{
Progress and Future Perspectives in Airborne Communication Networking
}

\author{
Kai-Daniel Büchter ${ }^{1}$ \\ ${ }^{1}$ Bauhaus Luftfahrt e.V., Willy-Messerschmitt-Str. 1, 82024 Taufkirchen, \\ kai-daniel.buechter@bauhaus-luftfahrt.net
}

\begin{abstract}
:
A simulation environment was built up to answer questions concerning the availability and performance of aeronautical ad-hoc networks (AAHN). The environment is able to consider basestations on the ground and satellite communication as internet gateways. Worldwide air traffic, with up to tens of thousands of flights per day can be considered in the simulations. For high data throughput, laser communication is envisioned for aircraft to aircraft communication and potentially also for gateway access. As for telemetry, hybridization is required for node localization and "bootstrapping" of connections. In this contribution, AAHN within a fleet of European aircraft are investigated in the context of air-to-ground and satellite infrastructure available today.
\end{abstract}

Key words: Aeronautical Ad-hoc Networks, laser communication, air-to-ground (A2G).

\section{Introduction}

Current trends in data-driven services in aviation (flight operations management, realtime system monitoring, in-flight connectivity etc.) increase the demands on communication systems, so that state-of-the-art satellite and air-to-ground (A2G) solutions with bitrates on the order of Mbit/s will be strained with regard to throughput, cost, and security in the near future. A complementary technology is freespace optical (FSO) communications, which offers tens of Gbps today (air-to-air / A2A, A2G) without occupying radio spectrum, and with inherent data tapping protection. The prospect of using FSO in the aeronautical context lies in stratospheric communication networks of aircraft or aeronautical ad-hoc networks (AAHN, e.g. [1] [2]), which could provide a throughput far beyond what is available today. Data transmission both in-between aircraft, and to the ground via multi-hop transmission, may be exploited for different types of applications and services. As of today (April 2018), the company Airborne Wireless Network [3] is striving to realize such a network. In this particular case, a hybrid RF-optical approach [4] is pursued; RF is required for signaling while the optical network is used for high-speed throughput. Hybridization may also provide link resilience and redundancy. Base-stations on the ground have also sprung up for mobile communication of civilian aircraft: Gogo has been providing $A 2 G$, in-flight internet in North America since 2008. Solutions based on 4G-technologies are currently being implemented, for example in Europe by Deutsche Telekom in cooperation with Inmarsat and Lufthansa (European Aviation Network or EAN [5]), and by SmartSky in the USA [6]. Around 300 EAN base-stations were set up across all EU member states by Telekom, with a communication radius of "typically" $90 \mathrm{~km}$ and bit rates of up to $75 \mathrm{Mbits} / \mathrm{s}$ to the aircraft. In this particular approach, ground connectivity, when available, augments $S$-band satellite communications whenever a fast connection is required. Adding AAHN "on top" could in this case increase the reach of the faster $A 2 G$ connections by multihop transmission, thereby enabling better distribution of available capacities. Therefore, in order to investigate $\mathrm{AAHN}$, several separate systems need to be considered: the air traffic system, the network infrastructure and topology, and the networking and transmission technologies. To this end, Bauhaus Luftfahrt has built up a corresponding simulation environment in order to investigate availability and performance metrics under fleet, infrastructure, and weather considerations. In this contribution, the potentials of $\mathrm{AAHN}$ are investigated considering a fleet of aircraft belonging to European airlines. Scenario-based results from the simulations are presented, considering a dense network of base-stations across Europe and the continental USA. 


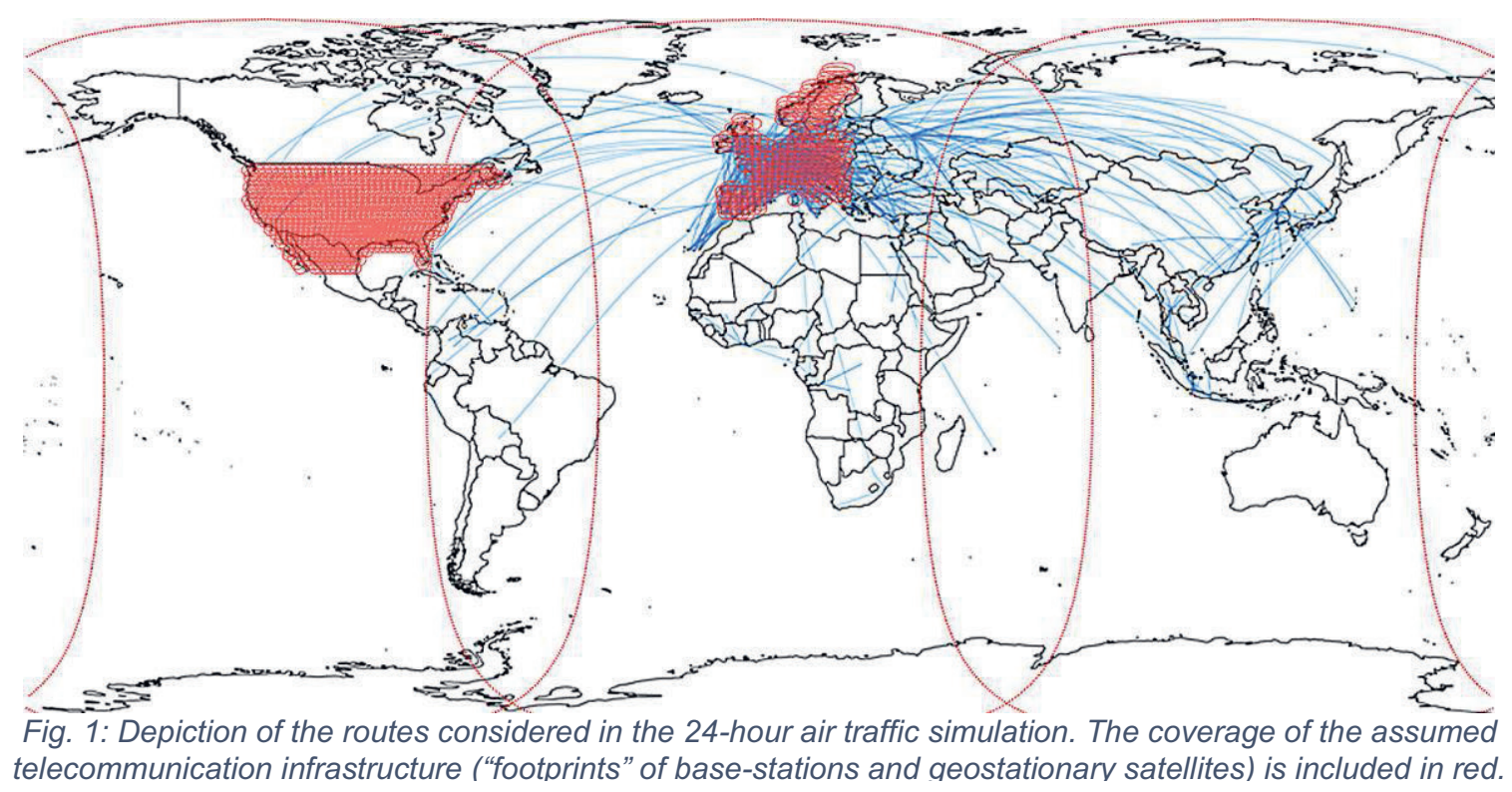

\section{Scenario Definition}

In the context of this contribution, a fleet of aircraft belonging to European airlines is considered and to this end, the 2016 edition of OAG flight schedule database [7] is used to simulate aircraft movements. The simulated day is October 29, 2016 - we selected the date before as an "average" day in worldwide air traffic [8]. Criteria for selecting flights for the simulations included whether the aircraft belonged to a European airline and whether it had more than 100 seats. Overall, the selection results in 16,862 flights considered overall, within a 24-hour timeframe. The respective routes are shown in Fig. 1.

\section{Infrastructure definition}

Base-stations on the ground and satellites are considered as internet gateways and the footprints of either are included in Fig. 1. We assume that base-stations are available across the EU and the USA. To this end, an algorithm places gateways over landmass in the range between $37^{\circ}$ and $75^{\circ}$ latitude, $-15^{\circ}$ and $21^{\circ}$ longitude (assumption for European infrastructure) and between $25^{\circ}$ and $48^{\circ}$ latitude, $-135^{\circ}$ and $-60^{\circ}$ degree longitude (assumption for US infrastructure). Based on available data on the EAN (about 300 stations covering 4.38 million $\mathrm{km}^{2}$ ), we estimate that the mean cell radius in Europe is $70 \mathrm{~km}$ and assume a maximum communication range of $150 \mathrm{~km}$. The available capacity per cell and antenna was set to $75 \mathrm{Mbit} / \mathrm{s}$ initially, or $225 \mathrm{Mbit} / \mathrm{s}$ per base-station, according to current specifications.

In addition to the base-stations, satellites are considered. For the sake of defining a scenario for a satellite capacity estimation, we assume $S$-band satellites with a transponder bandwidth of $15 \mathrm{MHz}$ in two polarizations. The overall throughput of a single satellite is then assumed to be channel capacity $\mathrm{x}$ polarization $\mathrm{x}$ number of spot beams. We assume that the satellite produces nine spot beams. In order to provide global coverage, we assume that three geostationary satellites are available.

\section{Estimation of communication system performance}

As different communication systems are involved, we discuss each type of connection next. An overview of our assumptions is given for A2A, A2G and A2S (air-to-satellite) connections in Tab. 1. Effectively, a 9-m dish and a $50-\mathrm{cm}$ effective aperture are assumed on the satellite and on the aircraft, respectively, for link-budget calculations. The assumed laser aperture is $10 \mathrm{~cm}$ in diameter. The number of A2A connections is limited to a maximum of four per aircraft. For the purpose of simplification, we consider unidirectional transmission in the simulation but assume that transmission performance is similar in both directions. 
Tab. 1: Definition of communication system performance for the AAHN simulations.

\begin{tabular}{|c|c|c|c|}
\hline Assumptions: & Air-to-air & Ground-to-air & Satellite-to-air \\
\hline Carrier wave & FSO: $1.67 \mu \mathrm{m}$ & $\mathrm{RF}: 2.1 \mathrm{GHz}$ & $\mathrm{RF}: 2.1 \mathrm{GHz}$ \\
\hline Bandwidth & $10 \mathrm{GHz}(\mathrm{FSO})$ & $15 \mathrm{MHz}$ & $15 \mathrm{MHz}$ \\
\hline $\begin{array}{c}\text { Antenna gain } \\
\text { (Tx / Rx) }\end{array}$ & $\begin{array}{c}2 \times 105 \mathrm{dBi} \\
=210 \mathrm{dBi}\end{array}$ & $\begin{array}{c}15 \mathrm{dBi}+3 \mathrm{dBi} \\
=18 \mathrm{dBi}\end{array}$ & $\begin{array}{c}46 \mathrm{dBi}+21 \mathrm{dBi} \\
=67 \mathrm{dBi}\end{array}$ \\
\hline Power & $27 \mathrm{dBm}(0.5 \mathrm{~W})$ & $46 \mathrm{dBm}(40 \mathrm{~W})$ & $51.5 \mathrm{dBm}(140 \mathrm{~W})$ \\
\hline $\begin{array}{c}\text { Additional losses } \\
\text { (Tx / Rx) }\end{array}$ & $2 \times 14 \mathrm{~dB}=28 \mathrm{~dB}$ & $2 \times 3 \mathrm{~dB}=6 \mathrm{~dB}$ & $2 \times 3 \mathrm{~dB}=6 \mathrm{~dB}$ \\
\hline $\begin{array}{c}\text { Atmospheric model } \\
\text { (clear sky } \\
\text { conditions) }\end{array}$ & {$[9]$} & $\begin{array}{c}\text { ITU model, } 58 \% \text { rel. } \\
\text { surface humidity }\end{array}$ & $\begin{array}{c}\text { ITU model, } 58 \% \text { rel. } \\
\text { surface humidity }\end{array}$ \\
\hline
\end{tabular}

According to [10], the feasible communication range for aircraft-to-aircraft communication systems using FSO is between 210 and $380 \mathrm{~km}$, depending on system considerations such as aperture diameter, mean power level and data rate. In practice, as clouds present obstacles to FSO systems, hybridization may be required as a means of mitigation. Our calculations indicate that, under clear-sky conditions, attenuation is small in comparison to free-space losses below $600 \mathrm{~km}$ A2A distance. Beyond $600 \mathrm{~km}$ aircraft separation, the beam passes through the troposphere and the effect of denser and more contaminated air (water content, aerosols, etc.) dominates overall losses. Earth curvature limits range geometrically beyond $750 \mathrm{~km}$, and clouds restrict practically achievable link lengths further.

Fig. 2, top shows the calculated Shannon capacity for the defined A2A link. In these calculations, the effect of fading losses (due to clear-air turbulence, beam-tracking errors, etc.) and high-altitude atmospheric contaminants (e.g. ice clouds) are not evaluated in detail. However, some system losses are assumed (beam coupling, return losses etc., cf. Tab. 1). Fig. 2, center shows calculated Shannon capacities for ground-to-air links. Assumptions regarding the FSO system are unchanged. As for RF, we assume an $S$-band carrier frequency of $2.1 \mathrm{GHz}$ with $15 \mathrm{MHz}$ of available bandwidth. The calculated Shannon capacity of approximately $100 \mathrm{Mbit} / \mathrm{s}$ at about $100 \mathrm{~km}$ distance compares well to EAN specification. In the FSO-case, communication range is less than half that of the A2A scenario and beyond $200 \mathrm{~km} \mathrm{A2G}$ distance, the performance of the optical link diminishes due to strong attenuation. It is clear that $A 2 G$ links enjoy less favorable conditions compared to A2A links.
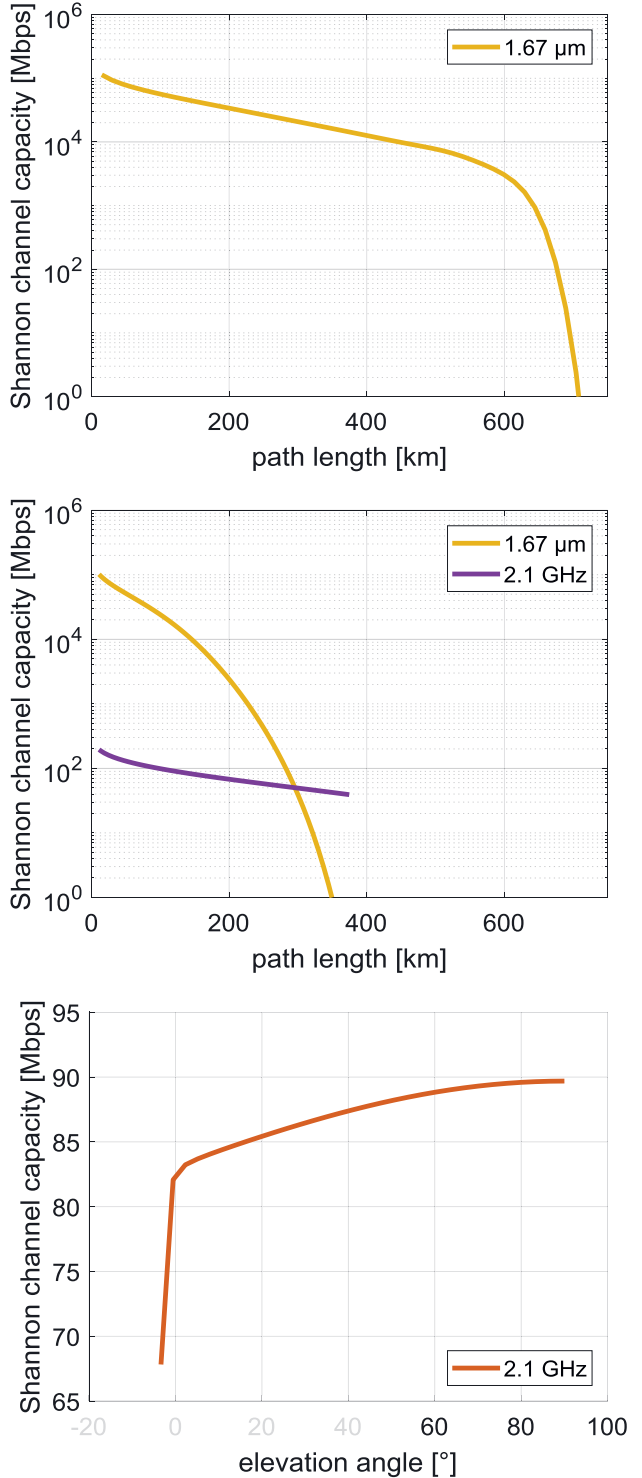

Fig. 2: Calculated Shannon capacities for transmission systems defined in Tab. 1, for $A 2 A$ (top), A2G (center) and A2S (bottom) connections. Dynamic effects (power fluctuations due to clear-air turbulence, beam tracking etc.) are disregarded. 
Tab. 2: Transmission system definition for initial simulations.

\begin{tabular}{|c|c|c|c|}
\hline Link & $\begin{array}{c}\text { Max. link } \\
\text { capacity }\end{array}$ & $\begin{array}{c}\text { Max. } \\
\text { throughput }\end{array}$ & $\begin{array}{c}\text { Max. } \\
\text { range }\end{array}$ \\
\hline A2A & $\begin{array}{c}1,000 \\
\text { Mbit/s }\end{array}$ & - & $\begin{array}{c}150-600 \\
\mathrm{~km}\end{array}$ \\
\hline $\mathrm{A} 2 \mathrm{G}$ & $75 \mathrm{Mbit} / \mathrm{s}$ & $225 \mathrm{Mbit} / \mathrm{s}$ & $150 \mathrm{~km}$ \\
\hline $\mathrm{A} 2 \mathrm{~S}$ & $80 \mathrm{Mbit} / \mathrm{s}$ & $1440 \mathrm{Mbit} / \mathrm{s}$ & N.A. \\
\hline
\end{tabular}

Obscurations by fog, clouds, aerosols and hydrosols as well as higher levels of turbulence restrict FSO availability. Therefore, in the simulations we assume that only RF links to the ground are available.

As for the S-band satellite link (Fig. 2, bottom), a maximum channel capacity of up to $90 \mathrm{Mbit} / \mathrm{s}$ is calculated, which we assume doubles with polarization diversity. From the scenario definition, the satellite throughput is defined as $1440 \mathrm{Mbit} / \mathrm{s}(2 \times 9 \times 80 \mathrm{Mbit} / \mathrm{s})$.

\section{Simulation Results: Contemporary Scenario}

Assumptions concerning the transmission systems for initial simulations are collected in Tab. 2. For the FSO system (A2A), we specify a capacity of $1 \mathrm{Gbit} / \mathrm{s}$, which is conservative for an optical link. However, as the A2A link is not the bottleneck in the scenario, a capacity increase does not affect the results in this scenario. As for the A2G link, $75 \mathrm{Mbit} / \mathrm{s}$ is assumed per cell according to specifications, with a maximum throughput of $225 \mathrm{Mbit} / \mathrm{s}$ per base-station (three cells). The maximum capacity for a single link is $75 \mathrm{Mbit} / \mathrm{s}$. As for the satellites, we assume that twice the calculated channel capacity is available per cell with polarization diversity, while the maximum throughput is proportional to the assumed number of beams. The capacity for a single A2S link is limited to $80 \mathrm{Mbit} / \mathrm{s}$. In our simulation model, we assume that the overall bandwidth per gateway (satellite or base-station) is distributed equally among the number of connected clusters. The number of aircraft in flight is shown in Fig. 3. Air traffic peaks at approximately 2800 aircraft. Typically, up to $20 \%$ of all aircraft are below clouds. The effect of considering clouds in the simulations is discussed later in the paper. The following results assume that clouds do not have an impact on link availability.

In Fig. 4, top, the percentage of connected aircraft and cluster size statistics are shown, including variances in cluster sizes. Without AAHN $\left(R_{\mathrm{AC}}=0 \mathrm{~km}\right), 45 \%$ of all flights are within range for $\mathrm{A} 2 \mathrm{G}$ communication on average. With increasing A2A-range, larger numbers of aircraft may participate in ad-hoc networking and the rate of aircraft with access to basestations also increases.

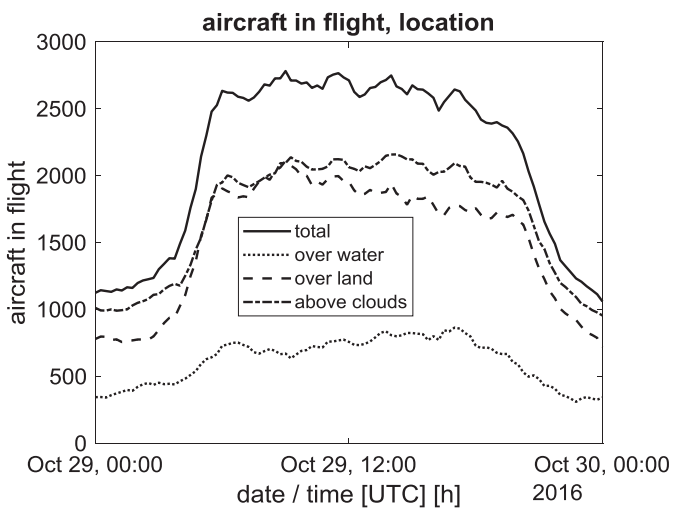

Fig. 3: Absolute number of aircraft in flight. The peak during daytime corresponds mainly to domestic flights.

Adding satellites provides nearly $100 \%$ connectivity to all aircraft. In addition, the variance in connectivity reduces with increasing communication range (Fig. 4, bottom: "scenarios" 1 to 10 with varying A2A-range and with or without satellites).
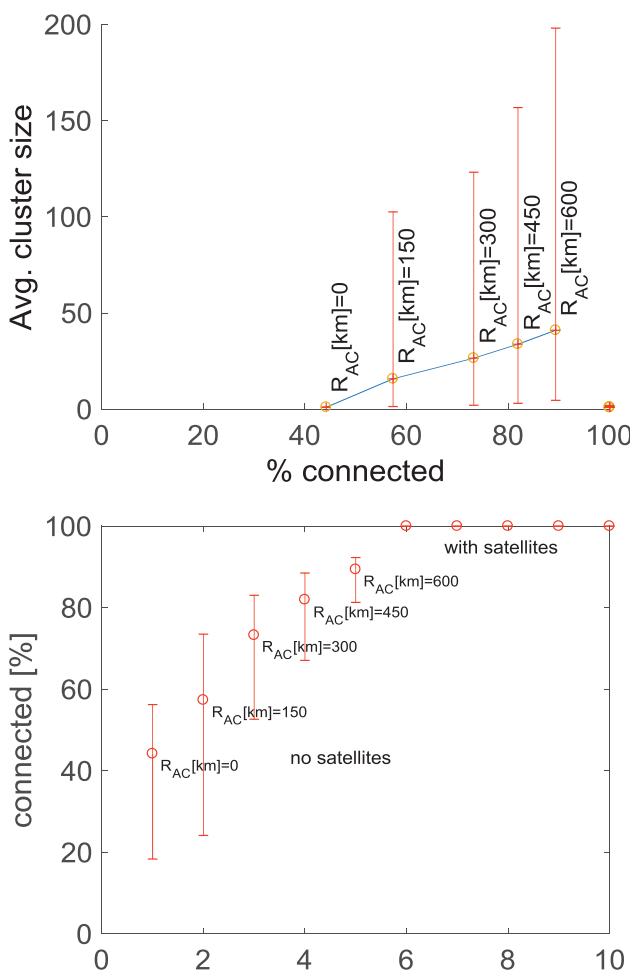

Fig. 4: Percentage of connected aircraft and cluster size statistics (top). Variance in connectivity for different A2A communication ranges (bottom). With satellites, all aircraft are practically always connected.

During the day, air traffic over Europe is dense and more than $50 \%$ of all aircraft in flight connect to the ground directly (Fig. 5, top). 
Adding A2A-links enables multi-hop connectivity to the ground via airborne networking, thereby enabling to off-load capacity from the satellites to the base-stations (Fig. 5, bottom). On average, around $40 \%$ of aircraft connect via AAHN exclusively in that case.
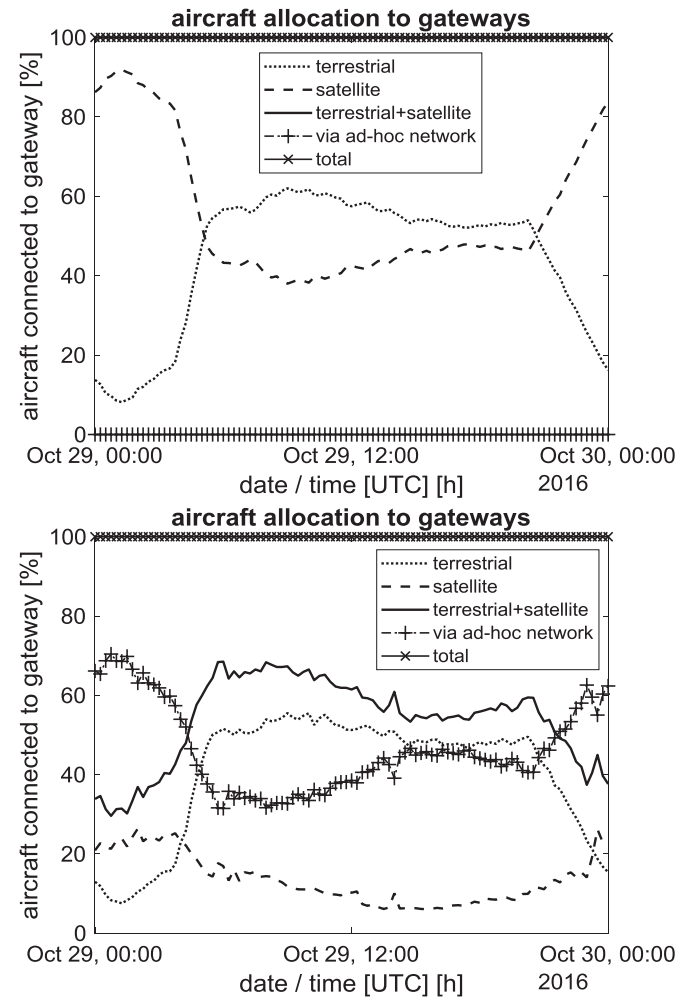

Fig. 5: The fraction of aircraft connected to either satellite or terrestrial gateways (without ad-hoc networking, top) and additionally via AAHN (bottom). In this scenario, especially satellite capacities are relieved as fewer aircraft depend on satellite connectivity $(300 \mathrm{~km}$ A2A-range assumed in this case). About half the aircraft may use AAHN for A2G connectivity via multi-hop transmission.

Statistics representing the maximum achievable, averaged throughput per aircraft are shown in Fig. 6. Throughput is calculated according to graph flow theory for each connected cluster of aircraft (which may have a size of one) and does not consider overhead due to protocols, retransmission of lost packets, etc. Moreover, it is assumed that each aircraft generates a demand of the full per-link capacity to "flood the network". The full per-link capacity to the ground is available to only a fraction of all aircraft, as all aircraft within a respective cell share capacity of the base-station without AAHN. With AAHN, the available infrastructure capacity is shared within the respective AAHNcluster.

In the scenario without airborne networking, about $50 \%$ of all flights enjoy bandwidths beyond a few Mbit/s according to the calculations. This seems reasonable, as roughly 1,000 aircraft have only satellite connectivity available during the air traffic peak (about $40 \%$ of 2,500 aircraft in-flight, Fig. 5). With a satellite throughput of $4,320 \mathrm{Mbit} / \mathrm{s}$ overall (three satellites with 1,440 Mbit/s each assumed), this results in about four Mbit/s per aircraft on average. Moreover, assuming that roughly 1,500 aircraft do have a ground connection (about $60 \%$ of all aircraft in-flight during peak hours), an averaged capacity per aircraft of $45 \mathrm{Mbit} / \mathrm{s} \quad(=300$ base-stations $\mathrm{x}$ $225 \mathrm{Mbit} / \mathrm{s} /$ 1,500 aircraft) would be expected for these flights, but considerable variance is evident.
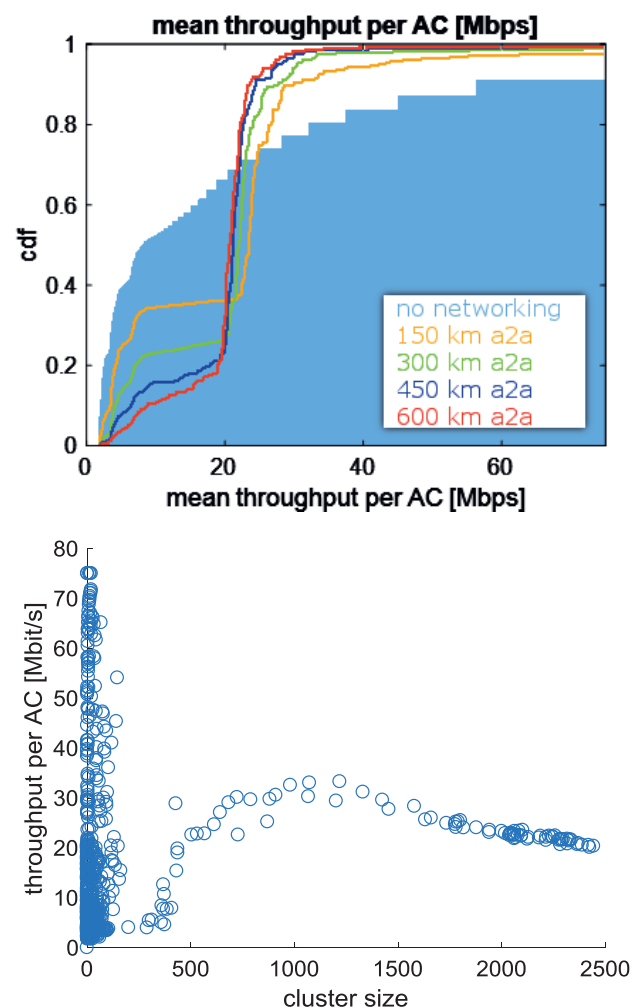

Fig. 6: Cumulative throughput statistics, averaged based on the maximum throughput per cluster of networked aircraft (top). Averaged capacity per aircraft as function of cluster size with $300 \mathrm{~km} \mathrm{A2A-}$ range (bottom).

When A2A networking is enabled by adding FSO connections, the participating aircraft may share the capacity within their respective mesh cluster, which leads to an equalizing effect in the statistics. Due to the large bandwidth of the FSO connection, the bottleneck to the internet is in the gateway connections. Throughput depends on cluster size and location: over Europe, the mean throughput per aircraft should average out according to air traffic density and cell density. Such an effect is suspected in Fig. 6, bottom: Above a cluster size of about 300 aircraft, the mean throughput peaks at a cluster size of around 1,100 aircraft, 
which is of the order of domestic flights over Europe during peak hours in the simulation. In the early morning hours and at night, however, a disproportionate number of flights either converge or diverge over Europe. Therefore, larger fractions of aircraft are beyond the boundaries of the area covered with basestations, so that fewer aircraft within the mesh provide ground connectivity.

Below a cluster size of 300 , the statistics include single aircraft and isolated clusters connected to ground or to satellite, especially beyond the borders of the European territory. Small clusters suffer from a higher volatility, because fewer connections are available with less redundancy but also, the full link capacity is available to some isolated aircraft and clusters.

\section{Simulation Results: Future Scenario}

In order to provide a future prospect, the simulation environment is modified in the following way: it is assumed that FSO connections are available to high altitude platforms (HAP) above the clouds. The communication range to HAPs $(\mathrm{A} 2 \mathrm{H})$ is set to $450 \mathrm{~km}$. As optical terminals are point-to-point connections, it is assumed that only a limited number of connections may be formed. Therefore, we assume that the HAPs have four optical connections each, with a capacity of $10 \mathrm{Gbit} / \mathrm{s}$ each and a corresponding downlink with $40 \mathrm{Gbit} / \mathrm{s}$. In addition, we assume that satellite laser terminals (SLT) are available to aircraft. Here, we assume that ten connections are available on three geostationary satellites each, with a capacity of $1 \mathrm{Gbit} / \mathrm{s}$. As for the aircraft, we assume a capacity of $10 \mathrm{Gbit} / \mathrm{s}$ and $300 \mathrm{~km}$ range. Furthermore, we limit demand per aircraft to $1 \mathrm{Gbit} / \mathrm{s}$, corresponding for example to a hundred video streams with 10Mbit/s each. An overview on the modified assumptions is given in Tab. 3 .

Tab. 3: Transmission system definition for future scenario.

\begin{tabular}{|c|c|c|c|}
\hline Link & $\begin{array}{c}\text { Max. link } \\
\text { capacity }\end{array}$ & $\begin{array}{c}\text { Max. } \\
\text { throughput }\end{array}$ & $\begin{array}{c}\text { Max. } \\
\text { range }\end{array}$ \\
\hline $\mathrm{A} 2 \mathrm{~A}$ & $10 \mathrm{Gbit} / \mathrm{s}$ & - & $300 \mathrm{~km}$ \\
\hline $\mathrm{A} 2 \mathrm{H}$ & $10 \mathrm{Gbit} / \mathrm{s}$ & $40 \mathrm{Gbit} / \mathrm{s}$ & $450 \mathrm{~km}$ \\
\hline $\mathrm{A} 2 \mathrm{~S}$ & $1 \mathrm{Gbit} / \mathrm{s}$ & $10 \mathrm{Gbit} / \mathrm{s}$ & N.A. \\
\hline
\end{tabular}

Simulation results are shown in Fig. 7. The fraction of aircraft which connect through the ad-hoc network is higher than before. Naturally, this is the case, as a limit was imposed on the number of gateway connections. Overall, on average about $10 \%$ of aircraft do not have a connection in this scenario - these could for example use RF satellite resources. However, nearly $80 \%$ of all aircraft have an averaged throughput beyond $200 \mathrm{Mbit} / \mathrm{s}$ and around $70 \%$ of all aircraft even have a throughput of more than $800 \mathrm{Mbit} / \mathrm{s}$.
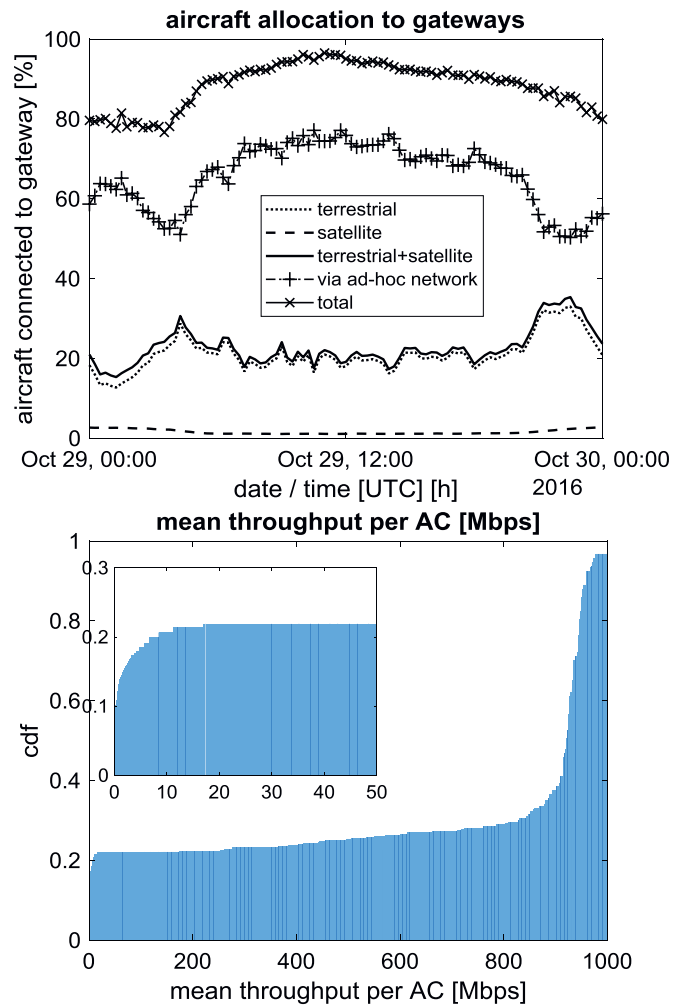

Fig. 7: Scenario with laser connections available between all nodes. As there is a limitation on the available satellite connections, full coverage is not achieved without additional RF satellite links, for example (top). Achievable, averaged bitrates for this scenario. The inset shows the lower left portion of the graph in detail. Around $70 \%$ of all aircraft see averaged bitrates of over $800 \mathrm{Mbit/s}$ (bottom).

Finally, when clouds are considered, based on NASA AIRS [11] satellite cloud top pressure data of the simulated day, overall availability suffers as seen in Fig. 8 (result from "future scenario"). In this case, links with a transmission path below or through the cloud top are blocked in the simulation. Chiefly, aircraft during the take-off and landing phases lose their connections. The fraction of aircraft connected directly to infrastructure changes only slightly, as the number of base- station terminals limits the number of connections in the scenario, but some of the gains of the AAHN are lost. Especially the connectivity during peak hours suffers - the main reason is that the frequent short-haul flights over Europe spend a considerable time during their take-off and landing phases below the cloud tops. Some of the losses should be recoverable by 
hybridization of the links. Fig. 8, bottom, shows the throughput per aircraft - as the link and infrastructure capacities are higher than the demand per aircraft, throughput approaches the set demand of $1 \mathrm{Gbit} / \mathrm{s}$ in this scenario, but doesn't peak as in Fig. 6, bottom.
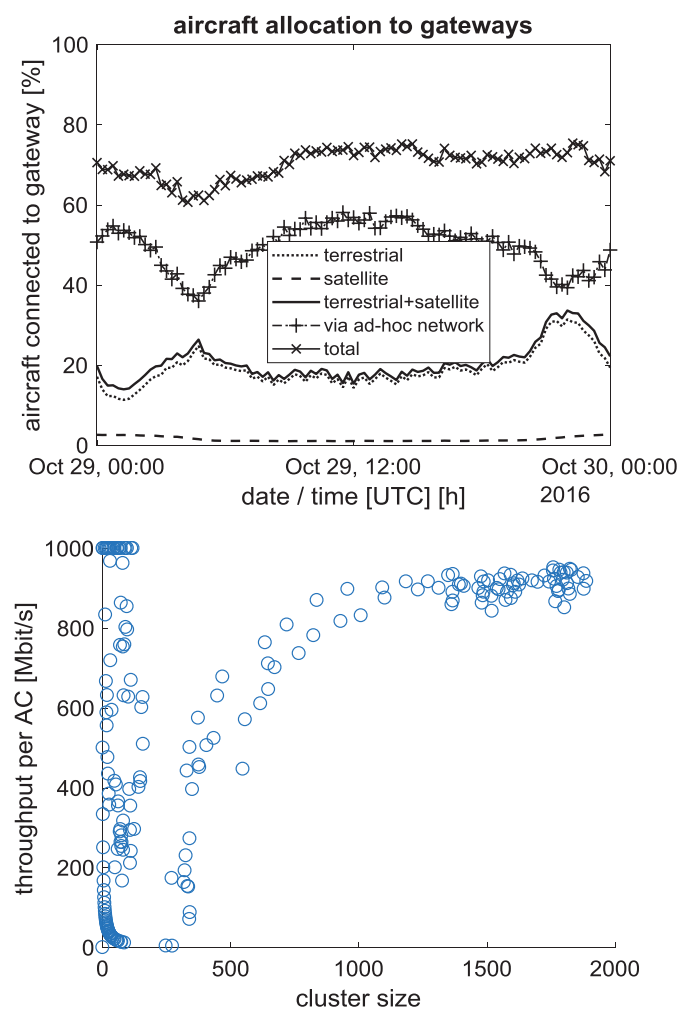

Fig. 8: Scenario above with added cloud top data which impede optical link availability. Mean throughput per aircraft as function of cluster-size is shown in the bottom graph.

\section{Summary and Conclusions}

In this contribution, the effect of adding ad-hoc networking capability to the commercial air traffic system was investigated within the context of ground infrastructure and satellite connectivity available today. Certain assumptions and calculations were made based especially on publicly available information on the European Aviation Network. We took certain liberties to represent, to our best knowledge, a feasible, near-future infrastructure perspective in our simulation environment. It was shown that the airborne network enables a redistribution of infrastructure capacities, such that the variance in throughput per aircraft is reduced and more aircraft can be provided with a moderate bandwidth. This is interpreted as a positive overall effect. Moreover, multi-hop communication to the ground frees up satellite capacities, which benefits aircraft without access to base-stations. Peak bandwidths per aircraft are reduced; however, this only affects a fraction of aircraft in-flight. With the infrastructure available today (as of 2018), adding aeronautical networking could, according to the simulations, provide an averaged throughput of at least $20 \mathrm{Mbits} / \mathrm{s}$ to up to $80 \%$ of all European aircraft, if these had a free-space optics-based networking capability (overhead not included).

In order to provide a future perspective, the simulation was modified to consider high-speed laser communication to high altitude platforms and satellites, with a limited number of available laser terminals per station, under certain assumptions concerning link capacity and range. Here it was calculated that an averaged throughput of $800 \mathrm{Mbits} / \mathrm{s}$ could be provided per aircraft. The total throughput is scalable with the number of gateway connections, demonstrating future growth potentials.

Future work may consider scenarios that evaluate the impact of adding high-throughput satellite connectivity (ku-/ka-band), for example, in a refined satellite model. From the perspective of business models, different fleets of airlines and aircraft can be considered with different communication capabilities and communication demands. For a defined fleet, the ratio of equipped aircraft can also be varied for phase-in studies. Adding flights to the simulated fleet improves AAHN availability, so business models should consider incentives for participation. Lastly, the data traffic models may be improved for example to evaluate the "goodput" in the network, and network dynamics may be evaluated for technical feasibility analyses, including for example link acquisition time and the impact on the network. Beyond internet connectivity, also the "intermesh" communication capacities may be of interest.

\section{References}

[1] D. Medina, F. Hoffmann, S. Ayaz und C.-H. Rokitansky, Feasibility of an Aeronautical Mobile Ad Hoc Network Over the North Atlantic Corridor, Proc. Fifth IEEE Conference on Sensor, Mesh, and Ad Hoc Communications and Networks (SECON), San Francisco, USA, 2008.

[2] B. Newton, J. Aikat und K. Jeffay, Concise Paper: Analysis of Topology Algorithms for Commercial Airborne Networks, IEEE 22nd International Conference on Network Protocols (ICNP), Research Triangle Park, USA, 2014.

[3] „Airborne Wireless Network," [Online] http://www.airbornewirelessnetwork.com/index. asp.

[4] L. B. Stotts, L. C. Andrews, P. C. Cherry, J. J. Foshee, P. J. Kolodzy, W. K. Mclntire, M. Northcott, R. L. Phillips, H. A. Pike, B. Stadler 
und D. W. Young, Hybrid Optical RF Airborne Communications, Proc. of the IEEE, Bd. 97, Nr. 6, pp. 1109-1127, 2009.

[5] Deutsche Telekom AG, 2015. [Online]. https://www.telekom.com/media/company/2882 50.

[6] „SmartSky Networks," [Online]. http://smartskynetworks.com/.

[7] „OAG Aviation Worldwide Limited,“ 2016. [Online]. http://www.oag.com/.

[8] K.-D. Büchter, Availability of Airborne Ad-hoc Communication Network in Global Air Traffic Simulation, 2016 10th International Symposium on Communication Systems, Networks and Digital Signal Processing (CSNDSP), Prague, 2016.

[9] L. Elterman, UV, Visible, and IR Attenuation for Altitudes to $50 \mathrm{~km}, 1968$, Environmental Research Papers, Nr. 285, 1968.

[10] H. Henninger und D. Giggenbach, Avionic Optical Links for High Data-rate Communications, in 25th International Congress of the Aeronautical Sciences, Hamburg, Germany, 2006.

[11] E. Fetzer, G. Hulley, E. Manning, J. Blaisdell, L. Iredell, J. Susskind, J. Warner, Z. Wei, W. Blackwell und E. Maddy, AIRS Version 6 Release Level 2 Product User Guide Version 1.1, E. T. Olson, Hrsg., Jet Propulsion Laboratory, Pasadena, CA: Jet Propulsion Laboratory, 2016. 